Remote Sens. 2010, 2, 2680-2699; doi:10.3390/rs2122680

OPEN ACCESS

Remote Sensing

ISSN 2072-4292

www.mdpi.com/journal/remotesensing

Article

\title{
Relationships Between Errors Propagated in Fraction of Vegetation Cover by Algorithms Based on a Two-Endmember Linear Mixture Model
}

\section{Kenta Obata and Hiroki Yoshioka *}

Department of Information Science and Technology, Aichi Prefectural University, 1522-3 Kumabari, Nagakute, Aichi, 480-1198, Japan; E-Mail: kenta.obata@ cis.aichi-pu.ac.jp

* Author to whom correspondence should be addressed; E-Mail: yoshioka@ ist.aichi-pu.ac.jp; Tel.:+81-0561-64-1111, ext. 3301.

Received: 3 November 2010; in revised form: 26 November 2010 / Accepted: 29 November 2010 / Published: 2 December 2010

\begin{abstract}
Remotely sensed reflectance spectra may be biased by several intervening factors, and the biases are propagated into estimations of the fraction of vegetation cover (FVC) by algorithms based on a linear mixture model (LMM). The errors propagated in FVCs depend on the retrieval algorithm used, due to differences in the assumptions of the model as well as constraints employed in the algorithm. These differences should be fully understood prior to algorithm selection for practical applications. Although numerous studies have investigated the relationships between errors propagated by different algorithms, these relationships have not been fully understood from a deterministic perspective. This study introduces a technique for deriving the analytical underpinnings of error propagation in FVC based on several LMM-based algorithms. The derivation assumes that measurement noise is band-correlated additive noise. The bias errors propagated in FVC depended on the endmember spectra assumed in the algorithm, the target spectrum, and the coefficients of the spectral vegetation index, which were employed as constraints, as well as magnitude of the input error. It was found that the relationships among the propagated errors assume asymmetric elliptical forms with coefficients that are determined by the input variables. These results suggest that the relationships depend heavily on the choice of endmember spectra as well as the spectrum of the target pixel and the vegetation index employed as a constraint. The present findings should assist in the selection of an optimum algorithm based on prior knowledge of the target field.
\end{abstract}


Keywords: fraction of vegetation cover; linear mixture model; propagated error; inter-algorithm relationship; vegetation index

\section{Introduction}

Land vegetation plays an important role in climatic, hydrologic, and geochemical cycles [1-3]. Thus, changes in the quantity and distribution of vegetation over land surfaces have a tremendous impact on the global climate. The status of land vegetation must be observed accurately to improve predictive climate models for carbon emission rates. Such a vegetated surface is often described precisely using biophysical parameters in a model [4]. One such parameter is the fraction of vegetation cover (FVC), which is a measure of the horizontal vegetation distribution, usually provided by satellite remote sensing data [4,5]. Information regarding the FVC is also considered to be an important indicator of forest degradation [6], land-use, land-cover change [7], and management or policy development [8]. Hence, accurate estimation of the FVC from remotely sensed data would contribute to a variety of environmental studies.

There are several approaches to retrieve the FVC from satellite data, for example, spectral unmixing of linear mixture models (LMM) [9-11], numerical inversion of radiative transfer models [12,13], and signal processing approaches, including neural networks [14,15]. Among these models, the first approach (LMM-based algorithm) is used most frequently due to its simplicity, rationality, and feasibility in practical applications.

The LMM-based algorithm permits some variations in implementing the model, which produces different FVC values, from a given target spectrum, via different algorithms. These variations arise from differences in the choice of endmember variable (which represents a pure spectral component) and the constraints imposed on the modeled spectrum in the algorithms. In a previous study [16], we derived the relationships among FVCs using three types of LMM-based algorithms, namely, reflectance-based LMM, vegetation index (VI)-based LMM, and isoline-based LMM. The first two algorithms differed in the identity of the endmember variable (reflectance or VI). The third algorithm differed from the other two in the choice of VI constraint. Information about the relationships among FVCs themselves is insufficient to inform the choice of an optimum algorithm, because the amplitude of the error due to measurement noise that is propagated in the FVC value should also vary among algorithms. To proceed one step further from our previous work, this study investigates the relationships among the errors propagated in FVCs using the three algorithms.

A remotely sensed reflectance spectrum suffers from some degree of uncertainty due to numerous intervening factors, such as instrumental noise, poor radiometric and geometric calibration, atmospheric influences, and soil background effects $[17,18]$. The errors in the spectral measurements should propagate into retrievals, and the amplitude of the propagated errors depends on the algorithm used. Several studies have investigated error propagation in fractional abundances retrieved using the reflectance-based LMM. Numerical simulations have been conducted to analyze the dependency of errors induced by uncertainties in the spectrophotometric calibration on the fraction of endmember components under the two-endmember assumption [19]. Similarly, the errors propagated into 
determination of the fractional abundance, induced by atmospheric contamination, were analyzed numerically using the multiple-endmember LMM [20]. The relationship between error-prone measurements (input) and fractional abundances (output) was derived analytically as a function of the standard deviation of the input error under the multiple-endmember assumption [21].

In this study, we focus on the errors propagated into FVC retrievals obtained from LMM-based algorithms. Although the relationships between FVCs by LMM-based algorithms have been examined [16], the relationships that describe the propagated errors have not been articulated. This information is necessary to guide selection of an optimum algorithm based on prior knowledge of the endmember spectra and variations in the range of target spectra within a field of study. This study contributes to this goal. Our objective is to derive the relationships that describe errors propagated into FVC retrievals computed using several LMM-based algorithms under the two-endmember assumption. We derive the relationship for errors propagated through all conceivable combinations of the algorithms within this framework.

\section{Background}

In the LMM, a target spectrum is represented by a linear sum of endmember spectra $[7,8,10,11]$. In this study, we limit both the number of endmembers and bands to two in order to facilitate an analytic discussion and to normalize the framework with respect to our previous discussion [16]. One of the endmember classes is vegetation, and the other is non-vegetation, usually represented by a soil spectrum. Hence, the weight for the vegetation endmember spectrum is the FVC value to be determined. The bands used in this study are in the red and near infrared (NIR), which are regions of the spectrum that are most frequently used for vegetation monitoring with respect to VI. Under these limitations, the modeled spectrum can be written in terms of the vegetation and the non-vegetation spectra $\left(\boldsymbol{\rho}_{v}=\left(\rho_{v, r}, \rho_{s, r}\right)\right.$ and $\boldsymbol{\rho}_{s}=\left(\rho_{s, r}, \rho_{s, n}\right)$, respectively), where the $\operatorname{FVC}(\hat{\omega})$ is a weight,

$$
\boldsymbol{\rho}_{m}(\hat{\omega})=\hat{\omega} \boldsymbol{\rho}_{v}+(1-\hat{\omega}) \boldsymbol{\rho}_{s} .
$$

Note that the unity constraint is imposed on the weights in the above equation. Below, we introduce three algorithms based on LMM under a two-endmember assumption. The details of these algorithms are provided in [16].

\subsection{Algorithm-1: Reflectance-Based LMM}

Algorithm-1 is called the reflectance-based LMM, and the value of the FVC $\left(\hat{\omega}_{1}\right)$ is determined from the minimization of the distance measure (often, the Euclidean norm) between the target spectrum, $\boldsymbol{\rho}_{t}=\left(\rho_{t, r}, \rho_{t, n}\right)$, and the modeled spectrum, $\boldsymbol{\rho}_{m}[10,11]$. The formulation of the FVC retrieval by this algorithm becomes

$$
\hat{\omega}_{1}=\frac{\boldsymbol{d} \cdot\left(\boldsymbol{\rho}_{t}-\boldsymbol{\rho}_{s}\right)}{\boldsymbol{d} \cdot \boldsymbol{d}}
$$

where $\boldsymbol{d}$ is the vector between $\boldsymbol{\rho}_{v}$ and $\boldsymbol{\rho}_{s}$ defined by

$$
\boldsymbol{d}=\boldsymbol{\rho}_{v}-\boldsymbol{\rho}_{s} .
$$


Table 1. Coefficients of the two-band VIs $\left(p_{i}, q_{i}\right.$, and $\left.r_{i}\right)$.

\begin{tabular}{ccccccc}
\hline & $p_{1}$ & $q_{1}$ & $r_{1}$ & $p_{2}$ & $q_{2}$ & $r_{2}$ \\
\hline NDVI & -1 & 1 & 0 & 1 & 1 & 0 \\
SAVI & $-(1+L)$ & $1+L$ & 0 & 1 & 1 & $L$ \\
EVI2 & -2.5 & 2.5 & 0 & 2.4 & 1 & 1 \\
\hline
\end{tabular}

\subsection{Algorithm-2: VI-Based LMM}

The second algorithm, algorithm-2, is a VI-based LMM that replaces reflectance in algorithm-1 with VI [3,22-27]. Numerous variations of the two-band VI $(v)$ have been proposed, and the function $f$ represents their model equation in the following general form,

$$
\begin{aligned}
v & =f(\boldsymbol{\rho}) \\
& =\frac{p_{1} \rho_{r}+q_{1} \rho_{n}+r_{1}}{p_{2} \rho_{r}+q_{2} \rho_{n}+r_{2}},
\end{aligned}
$$

where the coefficients $p_{i}, q_{i}$, and $r_{i}$ depend on the actual choice of VI. The function $f$ covers some of the well-known VIs, such as NDVI [28], SAVI [29], and EVI2 [30]. The coefficients for those VIs are summarized in Table 1 . The function $f$ can also be rewritten by

$$
f(\boldsymbol{\rho})=\frac{\boldsymbol{c}_{1} \cdot \boldsymbol{\rho}+r_{1}}{\boldsymbol{c}_{2} \cdot \boldsymbol{\rho}+r_{2}}
$$

where the two vectors represented by $c_{i}$ are defined by

$$
\boldsymbol{c}_{i}=\left(p_{i}, q_{i}\right), \quad(i=1,2) .
$$

Using the above definitions, the spectrum of a target pixel $\boldsymbol{\rho}_{t}$ can be transformed into the VI $\left(v_{t}\right)$ by

$$
v_{t}=f\left(\boldsymbol{\rho}_{t}\right) .
$$

We define the VI values of each endmember spectrum, $v_{v}$ and $v_{s}$, for the vegetation and non-vegetation endmember spectra, respectively,

$$
\begin{aligned}
& v_{v}=f\left(\boldsymbol{\rho}_{v}\right), \\
& v_{s}=f\left(\boldsymbol{\rho}_{s}\right) .
\end{aligned}
$$

The FVC value obtained from this algorithm $\left(\hat{\omega}_{2}\right)$ is written as

$$
\hat{\omega}_{2}=\frac{v_{t}-v_{s}}{v_{v}-v_{s}} .
$$

\subsection{Algorithm-3: Isoline-Based LMM}

The third algorithm, algorithm-3, is the isoline-based LMM, in which a target spectrum is modeled in the same way as in algorithm-1. The only difference between algorithms-1 and -3 is that in algorithm-3, 
the VI is used as a constraint to determine the FVC, similar to algorithm-2. For this reason, algorithm-3 can be viewed as a combination of algorithms-1 and -2 [31-33]. The FVC estimation by this algorithm $\left(\hat{\omega}_{3}\right)$ can be written (as a function of $v_{t}$ ) as

$$
\hat{\omega}_{3}=\frac{\left(\boldsymbol{c}_{1}-v_{t} \boldsymbol{c}_{2}\right) \cdot \boldsymbol{\rho}_{s}+r_{1}-v_{t} r_{2}}{\left(v_{t} \boldsymbol{c}_{2}-\boldsymbol{c}_{1}\right) \cdot \boldsymbol{d}}
$$

\subsection{Relationship Among the Estimated FVC Values Retrieved by the Three Algorithms}

The analytical functions that describe the three algorithms have been investigated by [16]. From that work, the FVCs estimated by algorithms-1 and -2 (and algorithms-1 and -3) are related by a one-to- $n$ mapping, meaning that an FVC value obtained from algorithm-1 cannot be uniquely transformed to an estimation computed using algorithm-2. Some degree of uncertainty is unavoidable in any transformation defined between these algorithms. In contrast, FVC estimations from algorithms- 2 and -3 have a one-to-one relationship because these algorithms are both functions of the target VI value, $v_{t}$. The relationship becomes

$$
\hat{\omega}_{3}=\frac{\hat{\omega}_{2}}{\nu \hat{\omega}_{2}+1-\nu}
$$

where

$$
\nu=\frac{\left(v_{v}-v_{s} \boldsymbol{c}_{2}\right) \cdot \boldsymbol{d}}{\left(v_{v} \boldsymbol{c}_{2}-\boldsymbol{c}_{1}\right) \cdot \boldsymbol{d}}
$$

This result indicates that an FVC provided by one of the algorithms can be uniquely transformed into an FVC value provided by the other. Note that Equation (12) is only applicable to cases in which the same VI model is assumed in both algorithms. If the algorithms assume different VIs, the relationship is no longer one-to-one.

\section{Derivation of the Propagated Error in FVC}

Spectral measurements from satellite sensors are degraded by several intervening factors. Measurement noise inevitably propagates into the estimated FVC. Below, we will derive the error propagated as a function of an input error, for each algorithm.

\subsection{Assumptions Applied to the Measurement Error and Error Propagation During Calculation of the FVC}

The uncertainties in the target spectrum are represented as a two-parameter normal distribution with standard deviation $\sigma_{t}$. The additive noise is assumed to be centered at a target reflectance spectrum in the red-NIR reflectance subspace, as illustrated in Figure 1. This representation has been used to model the synthetic error in measurements, for example, the instrumental noise, sensor calibration deficiencies, and atmospheric effects [34]. FVCs are computed from a normally distributed reflectance spectra around the true target spectrum in each algorithm. The computed FVC distribution can be described as a transformation of this normal distribution into FVC values, which are then distributed over the modeled subspace in reflectance space (Figure 1). As an example, Figure 1 illustrates the 
Figure 1. Two-parameter normal distribution of the input noise centered on a target spectrum, and FVC distributions estimated using two algorithms. The two-dimensional distribution is projected onto the modeled subspace spanned by the two endmember spectra in the red-NIR reflectance space. The FVC distributions estimated using each algorithm are denoted by red and blue lines, along with the line between the two endmember spectra.

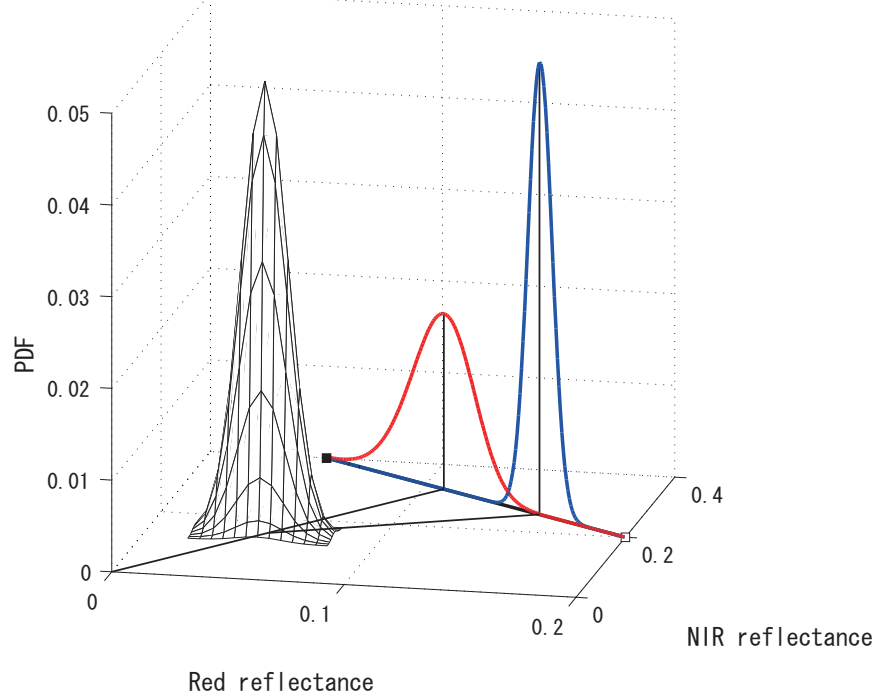

result of transforming a normal distribution onto FVC space using algorithms-1 and -3 with NDVI. The FVC distributions estimated by these algorithms show large discrepancies, indicative of the differences between the algorithms. The discrepancies arise not only from the FVC itself, but also from the standard deviations of the FVC distributions produced by each algorithm. An analytical description of the relationship between the standard deviations (amplitudes of the propagated error) is the center of our discussion. In this study, we focus on the difference from mean FVC value as a function of $\sigma_{t}$.

The variable $\sigma_{t}$ can be considered as representing the magnitude of the uncertainty, i.e., the radius of a circle circumscribing the target spectrum (Figure 2). We represent the reflectance spectra on the circle by a directional vector (unit vector) $e$,

$$
\boldsymbol{e}=(\cos \theta, \sin \theta)
$$

Using the above representation, a biased target spectrum, $\rho_{t, \epsilon}$ may be written by

$$
\boldsymbol{\rho}_{t, \epsilon}=\boldsymbol{\rho}_{t}+\sigma_{t} \boldsymbol{e}
$$

The above expression is equivalent to a measured spectrum that includes band-correlated additive noise described by the fixed values $\sigma_{t}$ and $\theta$ (Figure 2).

\subsection{Error Propagated in the FVC Using Algorithm-1}

The FVC estimation from algorithm-1, with the propagated error $\left(\hat{\omega}_{1, \epsilon}\right)$, may be written as

$$
\hat{\omega}_{1, \epsilon}=\frac{\boldsymbol{d} \cdot\left(\boldsymbol{\rho}_{t, \epsilon}-\boldsymbol{\rho}_{s}\right)}{\boldsymbol{d} \cdot \boldsymbol{d}} .
$$


Figure 2. Illustration of the noise added to an assumed target spectrum, used to derive the noise propagation function. The input noise is modeled as band-correlated additive noise. The angular parameter $\theta$ determines the direction of the band-correlation $e$. The magnitude of the additive noise is represented by a parameter $\sigma_{t}$, which is equivalent to the standard deviation of the two-parameter normal distribution describing the band-uncorrelated noise.

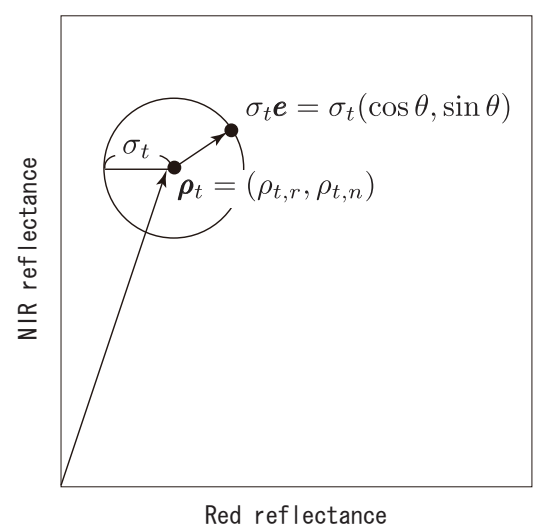

We assume that the propagated error in the FVC may be modeled using an additive form. Rewriting Equation (16) by explicitly expressing the error propagated in the FVC estimation by algorithm-1, $\epsilon_{1}$, yields

$$
\hat{\omega}_{1, \epsilon}=\hat{\omega}_{1}+\epsilon_{1}
$$

and we can solve for $\epsilon_{1}$ by subtracting Equations (2) from (16),

$$
\epsilon_{1}=\hat{\omega}_{1, \epsilon}-\hat{\omega}_{1}=\frac{\sigma_{t}}{\boldsymbol{d} \cdot \boldsymbol{d}} \boldsymbol{d} \cdot \boldsymbol{e} .
$$

\subsection{Error Propagated in the FVC Using Algorithm-2}

FVC retrieval using algorithm-2, with propagated error $\hat{\omega}_{2, \epsilon}$, is written by

$$
\hat{\omega}_{2, \epsilon}=\frac{v_{t, \epsilon}-v_{s}}{v_{v}-v_{s}},
$$

where $v_{t, \epsilon}$ is the VI value for $\boldsymbol{\rho}_{t, \epsilon}$, which is defined by

$$
v_{t, \epsilon}=\frac{\boldsymbol{c}_{1} \cdot \boldsymbol{\rho}_{t, \epsilon}+r_{1}}{\boldsymbol{c}_{2} \cdot \boldsymbol{\rho}_{t, \epsilon}+r_{2}}
$$

As in the previous subsection, $\hat{\omega}_{2, \epsilon}$ is assumed to be expressible as a sum of $\hat{\omega}_{2}$ and the error propagated in the estimation of FVC using algorithm-2, $\epsilon_{2}$,

$$
\hat{\omega}_{2, \epsilon}=\hat{\omega}_{2}+\epsilon_{2}
$$

Subtracting Equation (10) from (19), we obtain

$$
\epsilon_{2}=\hat{\omega}_{2, \epsilon}-\hat{\omega}_{2}=\frac{\sigma_{t} \boldsymbol{a} \cdot \boldsymbol{e}}{\sigma_{t} \boldsymbol{b} \cdot \boldsymbol{e}+\phi}
$$


where

$$
\begin{aligned}
\phi & =\left(v_{v}-v_{s}\right)\left(\boldsymbol{c}_{2} \cdot \boldsymbol{\rho}_{t}+r_{2}\right)^{2}, \\
\boldsymbol{a} & =\left(a_{1}, a_{2}\right)=\left(\boldsymbol{c}_{2} \cdot \boldsymbol{\rho}_{t}+r_{2}\right) \boldsymbol{c}_{1}-\left(\boldsymbol{c}_{1} \cdot \boldsymbol{\rho}_{t}+r_{1}\right) \boldsymbol{c}_{2}, \\
\boldsymbol{b} & =\left(b_{1}, b_{2}\right)=\left(v_{v}-v_{s}\right)\left(\boldsymbol{c}_{2} \cdot \boldsymbol{\rho}_{t}+r_{2}\right) \boldsymbol{c}_{2} .
\end{aligned}
$$

\subsection{Error Propagated in the FVC Using Algorithm-3}

The biased FVC value obtained from algorithm-3 $\left(\hat{\omega}_{3, \epsilon}\right)$ is written as

$$
\hat{\omega}_{3, \epsilon}=\frac{\left(\boldsymbol{c}_{1}-v_{t, \epsilon} \boldsymbol{c}_{2}\right) \cdot \boldsymbol{\rho}_{s}+r_{1}-v_{t, \epsilon} r_{2}}{\left(v_{t, \epsilon} \boldsymbol{c}_{2}-\boldsymbol{c}_{1}\right) \cdot \boldsymbol{d}}
$$

Equations (11) and (26) may be rewritten to facilitate the analytical discussion as follows

$$
\begin{gathered}
\hat{\omega}_{3}=\gamma+\frac{\zeta}{v_{t}+\eta}, \\
\hat{\omega}_{3, \epsilon}=\gamma+\frac{\zeta}{v_{t, \epsilon}+\eta}
\end{gathered}
$$

where

$$
\begin{aligned}
& \gamma=-\frac{\boldsymbol{c}_{2} \cdot \boldsymbol{\rho}_{s}+r_{2}}{\boldsymbol{c}_{2} \cdot \boldsymbol{d}}, \\
& \eta=-\frac{\boldsymbol{d} \cdot \boldsymbol{c}_{1}}{\boldsymbol{d} \cdot \boldsymbol{c}_{2}} \\
& \zeta=\frac{\left[\left(\boldsymbol{c}_{1} \cdot \boldsymbol{\rho}_{s}+r_{1}\right) \boldsymbol{c}_{2}-\left(\boldsymbol{c}_{2} \cdot \boldsymbol{\rho}_{s}+r_{2}\right) \boldsymbol{c}_{1}\right] \cdot \boldsymbol{\rho}_{v}+\left(r_{2} \boldsymbol{c}_{1}-r_{1} \boldsymbol{c}_{2}\right) \cdot \boldsymbol{\rho}_{s}}{\left(\boldsymbol{c}_{2} \cdot \boldsymbol{d}\right)^{2}} .
\end{aligned}
$$

From Equations (27) and (28), the propagated error $\epsilon_{3}$ becomes

$$
\epsilon_{3}=\hat{\omega}_{3, \epsilon}-\hat{\omega}_{3}=\frac{\sigma_{t} s \cdot \boldsymbol{e}}{\sigma_{t} \boldsymbol{t} \cdot \boldsymbol{e}+\psi},
$$

where

$$
\begin{aligned}
\psi & =\left[\boldsymbol{c}_{1} \cdot \boldsymbol{\rho}_{t}+r_{1}+\eta\left(\boldsymbol{c}_{2} \cdot \boldsymbol{\rho}_{t}+r_{2}\right)\right]^{2} \\
\boldsymbol{s} & =\zeta\left[\left(\boldsymbol{c}_{1} \cdot \boldsymbol{\rho}_{t}+r_{1}\right) \boldsymbol{c}_{2}-\left(\boldsymbol{c}_{2} \cdot \boldsymbol{\rho}_{t}+r_{2}\right) \boldsymbol{c}_{1}\right], \\
\boldsymbol{t} & =\left(\boldsymbol{c}_{1}+\eta \boldsymbol{c}_{2}\right)\left[\boldsymbol{c}_{1} \cdot \boldsymbol{\rho}_{t}+r_{1}+\eta\left(\boldsymbol{c}_{2} \cdot \boldsymbol{\rho}_{t}+r_{2}\right)\right]
\end{aligned}
$$

In the next section, we plot the relationships among $\epsilon_{1}, \epsilon_{2}$, and $\epsilon_{3}$, obtained by numerical simulation.

\section{Numerical Simulations of the Inter-Algorithm Relationships}

Before we derive the relationships among the propagated errors analytically, we present numerical examples of the relationships determined by simulation. These examples provide an empirical starting point for describing the relationships. The simulations were conducted, assuming that vegetation and non-vegetation endmember spectra in the red-NIR reflectance subspace could be described by $(0.05,0.4)$ and $(0.2,0.2)$, respectively. Three target spectra were assumed: A, $(0.1,0.2)$; B $(0.06,0.25)$; and 
C $(0.25,0.33)$, as illustrated in Figure 3(a). Note that the solid line in Figure 3(a) represents the model subspace of the LMM retrieval algorithms, which is the focus of this study. Band-correlated additive noise was then introduced into the three target spectra represented by $\sigma_{t} e$ in Equation (15). The magnitude of the noise, $\sigma_{t}$ was set to 0.01 during the simulations. The errors propagated in the FVC using the three algorithms $\left(\epsilon_{1}, \epsilon_{2}\right.$, and $\left.\epsilon_{3}\right)$ were first computed by varying the parameter $\theta$ in Equations (18), (22), and (32). The relationships among $\epsilon_{1}, \epsilon_{2}$, and $\epsilon_{3}$ were obtained numerically and are plotted in Figure 3(b-d).

From Figure 3(b,c), the relationships between errors propagated in algorithm-1 and errors propagated in algorithms-2 and -3 tended to be elliptical on all target spectra. In contrast, Figure 3(d) shows the existence of a one-to-one relationship between the errors propagated in algorithms- 2 and -3 , in each spectrum, which was expected from our previous work [16]. In the following sections, we derive these relationships analytically for all possible combinations of inter- and intra-algorithm relationships. A schematic diagram describing all algorithm combinations is shown in Figure 4.

\section{Inter-Algorithm Relationships of the Propagated Error}

In this section, we derive the inter-algorithm relationships describing the errors propagated in FVC estimations, assuming the same VI model for algorithms-2 and -3 . There were no differences in the VI constraints among the retrieval algorithms.

\subsection{Relationship Between Algorithms-1 and -2}

The errors propagated by algorithms- 1 and -2 can be related by eliminating the parameter $\theta$ from the representations of $\epsilon_{1}$ and $\epsilon_{2}$ (Equations (18) and (22)). These two equations can be written in matrix form as

$$
\sigma_{t} M e=m
$$

where

$$
\begin{aligned}
& \boldsymbol{M}=\left[\begin{array}{cc}
\rho_{v, r}-\rho_{s, r} & \rho_{v, n}-\rho_{s, n} \\
b_{1} \epsilon_{2}-a_{1} & b_{2} \epsilon_{2}-a_{2}
\end{array}\right], \\
& \boldsymbol{m}=\left[\begin{array}{c}
(\boldsymbol{d} \cdot \boldsymbol{d}) \epsilon_{1} \\
-\phi \epsilon_{2}
\end{array}\right] .
\end{aligned}
$$

Solving Equation (36) for $e$, we have

$$
e=\frac{1}{\sigma_{t}} M^{-1} m
$$

where

$$
\begin{aligned}
\boldsymbol{M}^{-1} & =\frac{1}{|\boldsymbol{M}|}\left[\begin{array}{cc}
b_{2} \epsilon_{2}-a_{2} & -\rho_{v, n}+\rho_{s, n} \\
-b_{1} \epsilon_{2}+a_{1} & \rho_{v, r}-\rho_{s, r}
\end{array}\right], \\
|\boldsymbol{M}| & =|\boldsymbol{d} \boldsymbol{b}| \epsilon_{2}-|\boldsymbol{d} \boldsymbol{a}| .
\end{aligned}
$$


Figure 3. Relationships among the errors propagated in each of the three algorithms, obtained by numerical simulation. (a) Plots of the endmember spectra and the three target spectra used in the simulation. The target spectra A, B, and C are indicated by blue, green, and red circles, respectively, in (a). Vegetation and non-vegetation endmember spectra are denoted by the filled and empty squares, respectively. The solid line indicates the subspace modeled by the reflectance-based LMM. (b) Cross plot of the propagated error using algorithms-1 $\left(\epsilon_{1}\right)$ and -2 $\left(\epsilon_{2}\right)$. The relationships among errors propagated in the three target spectra, obtained by simulation, are represented by the lines of the corresponding color, blue, green, and red, for A, B, and C, respectively. Similarly, (c) and (d) are the relationships between algorithms-1 $\left(\epsilon_{1}\right)$ and $-3\left(\epsilon_{3}\right)$, and between algorithms-2 $\left(\epsilon_{2}\right)$ and $-3\left(\epsilon_{3}\right)$, respectively. Note that NDVI was chosen as the conditions or constraints used in algorithms-2 and -3 for those simulations.

(a)

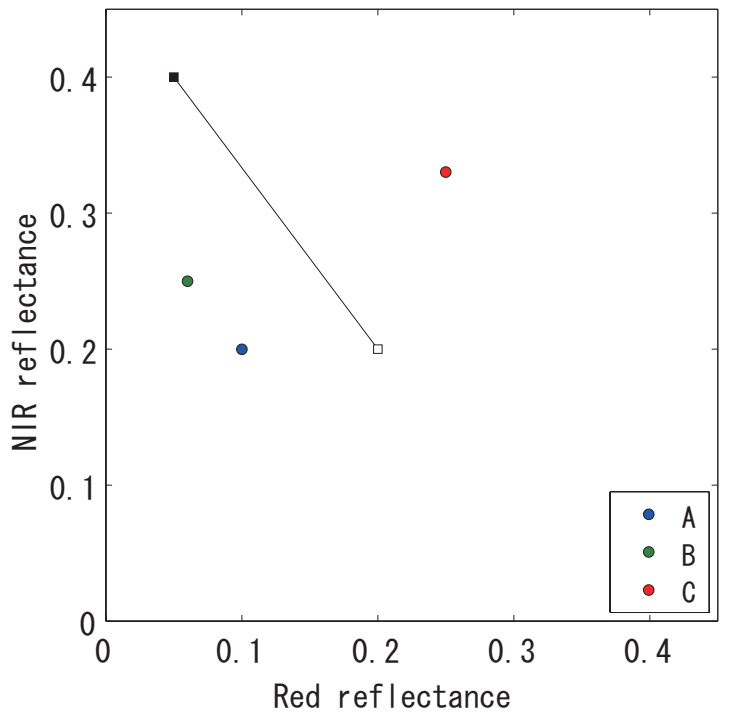

(c)

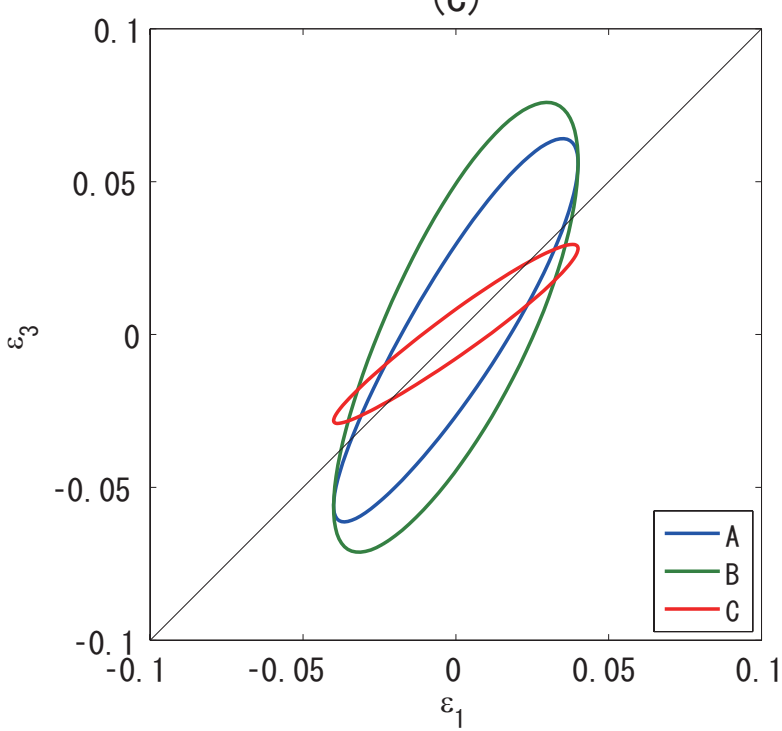

(b)

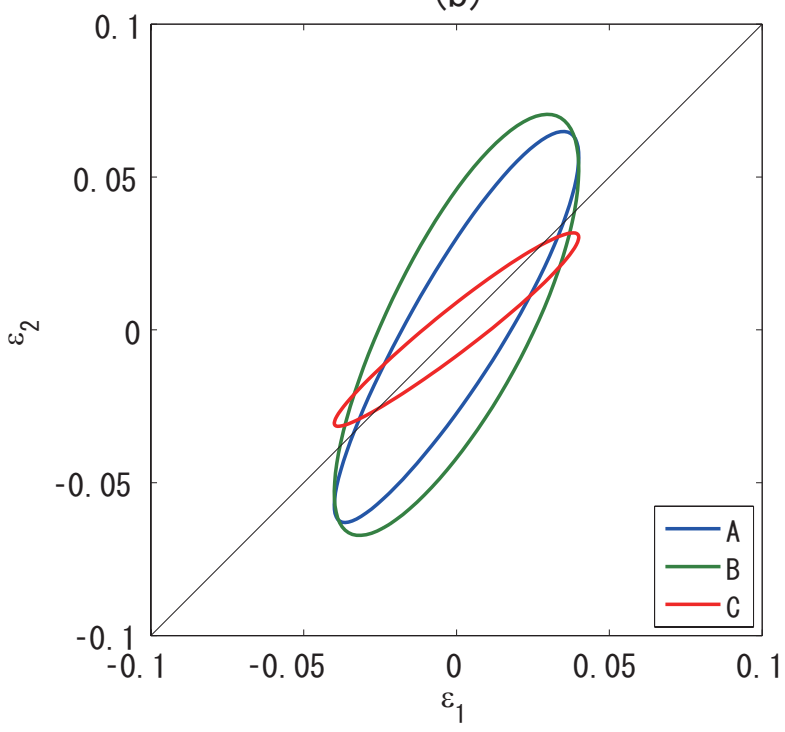

(d)

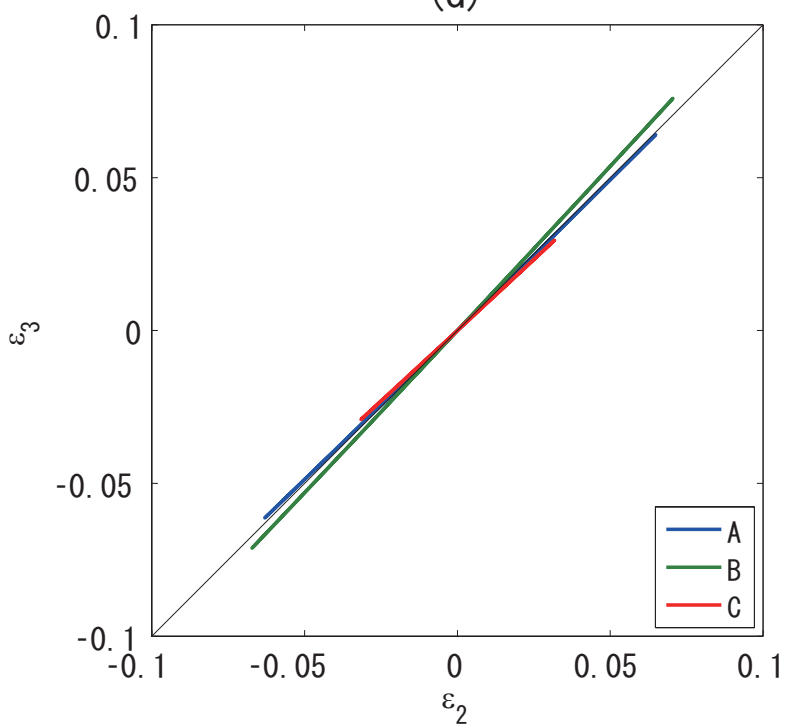


Figure 4. Schematic diagram describing the relationships among errors propagated in each algorithm considered in this study. The relationships between the errors propagated by each pair of algorithms are indicated by the colored bidirectional arrows. Information about the corresponding sections and equations is also provided in the figure.

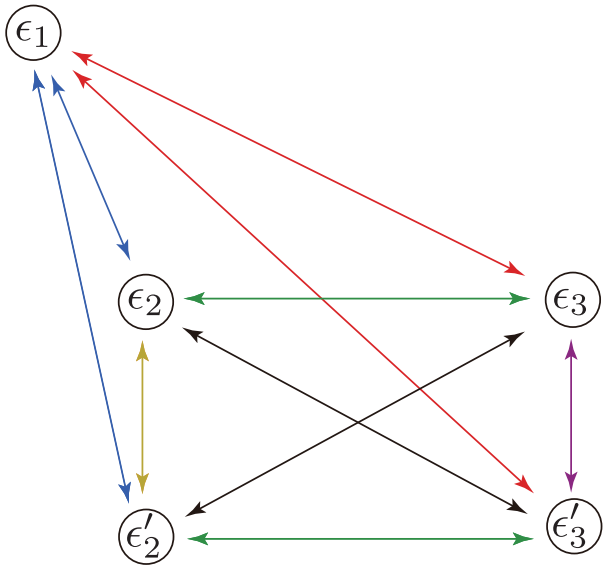

(1) Propagated error by algorithm-1

(є2) Propagated error by algorithm-2 with VI-1

$\epsilon_{2}^{\prime}$ Propagated error by algorithm-2 with VI-2

(€3) Propagated error by algorithm-3 with VI-1

$\epsilon_{3}^{\prime}$ Propagated error by algorithm-3 with VI-2

$\longleftrightarrow$ Sec.5 Eq. (44) $\longleftrightarrow$ Sec.6 Eq. (66)

$\longleftrightarrow$ Sec.5 Eq. (46) $\longleftrightarrow$ Sec.6 Eq. (75)

$\longleftrightarrow$ Sec.5 Eq. $(51) \longleftrightarrow$ Sec.7 Eq. (79)

Equation (39) can also be written by

$$
\boldsymbol{e}=\frac{1}{\sigma_{t}|\boldsymbol{M}|}\left[\begin{array}{c}
b_{2}(\boldsymbol{d} \cdot \boldsymbol{d}) \epsilon_{1} \epsilon_{2}-a_{2}(\boldsymbol{d} \cdot \boldsymbol{d}) \epsilon_{1}+\phi\left(\rho_{v, n}-\rho_{s, n}\right) \epsilon_{2} \\
-b_{1}(\boldsymbol{d} \cdot \boldsymbol{d}) \epsilon_{1} \epsilon_{2}+a_{1}(\boldsymbol{d} \cdot \boldsymbol{d}) \epsilon_{1}-\phi\left(\rho_{v, r}-\rho_{s, r}\right) \epsilon_{2}
\end{array}\right]
$$

To eliminate $\theta$, which is included implicitly in $e$, we use the Pythagorean theorem,

$$
\boldsymbol{e} \cdot \boldsymbol{e}=\sin ^{2} \theta+\cos ^{2} \theta=1 .
$$

Substituting Equation (42) into Equation (43), we obtain the relationship between $\epsilon_{1}$ and $\epsilon_{2}$,

$$
P_{1} \epsilon_{1}^{2} \epsilon_{2}^{2}+P_{2} \epsilon_{1}^{2} \epsilon_{2}+P_{3} \epsilon_{1} \epsilon_{2}^{2}+P_{4} \epsilon_{1}^{2}+P_{5} \epsilon_{2}^{2}+P_{6} \epsilon_{1} \epsilon_{2}+P_{7} \epsilon_{2}+P_{8}=0
$$

where

$$
\begin{aligned}
& P_{1}=(\boldsymbol{d} \cdot \boldsymbol{d})^{2}(\boldsymbol{b} \cdot \boldsymbol{b}), \\
& P_{2}=-2(\boldsymbol{d} \cdot \boldsymbol{d})^{2}(\boldsymbol{a} \cdot \boldsymbol{b}), \\
& P_{3}=2 \phi(\boldsymbol{d} \cdot \boldsymbol{d})(\boldsymbol{d} \cdot \boldsymbol{b}), \\
& P_{4}=(\boldsymbol{d} \cdot \boldsymbol{d})^{2}(\boldsymbol{a} \cdot \boldsymbol{a}), \\
& P_{5}=\phi^{2}(\boldsymbol{d} \cdot \boldsymbol{d})-\sigma_{t}^{2}|\boldsymbol{d} \boldsymbol{b}|^{2}, \\
& P_{6}=-2 \phi(\boldsymbol{d} \cdot \boldsymbol{d})(\boldsymbol{d} \cdot \boldsymbol{a}), \\
& P_{7}=2 \sigma_{t}^{2}|\boldsymbol{d} \boldsymbol{b}||\boldsymbol{d} \boldsymbol{a}|, \\
& P_{8}=-\sigma_{t}^{2}|\boldsymbol{d} \boldsymbol{a}|^{2} .
\end{aligned}
$$

The relationship between the errors propagated by algorithms- 1 and -2 can be expressed using a fourth-degree polynomial. The maximum degree term is the product of the square of the two propagated errors. The coefficients of the polynomial $\left(P_{i}, i=1,2, \cdots, 8\right)$ depend on the target spectrum, 
endmember spectra, and the choice of the VI model used in algorithm-2 (below, these variables are called the input data.), in addition to magnitude of the input noise. Equation (44) represents an asymmetric ellipse, the shape of which depends on the coefficients $P_{i}$. This result agrees with the numerical experiments (elliptical relationships) shown in Figure 3(b).

\subsection{Relationship Between Algorithms-1 and -3}

The relationship between errors propagated by algorithms- 1 and -3 can be derived using the same steps taken in the previous subsection. By noting the similarities of the representations between algorithms- 1 and -2 , we simply substitute $\boldsymbol{a}, \boldsymbol{b}$, and $\phi$ with $\boldsymbol{s}, \boldsymbol{t}$, and $\psi$, respectively, to obtain the final form. The relationship between $\epsilon_{1}$ and $\epsilon_{3}$ results in the following form,

$$
Q_{1} \epsilon_{1}^{2} \epsilon_{3}^{2}+Q_{2} \epsilon_{1}^{2} \epsilon_{3}+Q_{3} \epsilon_{1} \epsilon_{3}^{2}+Q_{4} \epsilon_{1}^{2}+Q_{5} \epsilon_{3}^{2}+Q_{6} \epsilon_{1} \epsilon_{3}+Q_{7} \epsilon_{3}+Q_{8}=0
$$

where

$$
\begin{aligned}
& Q_{1}=(\boldsymbol{d} \cdot \boldsymbol{d})^{2}(\boldsymbol{t} \cdot \boldsymbol{t}), \\
& Q_{2}=-2(\boldsymbol{d} \cdot \boldsymbol{d})^{2}(\boldsymbol{s} \cdot \boldsymbol{t}), \\
& Q_{3}=2 \psi(\boldsymbol{d} \cdot \boldsymbol{d})(\boldsymbol{d} \cdot \boldsymbol{t}), \\
& Q_{4}=(\boldsymbol{d} \cdot \boldsymbol{d})^{2}(\boldsymbol{s} \cdot \boldsymbol{s}), \\
& Q_{5}=\psi^{2}(\boldsymbol{d} \cdot \boldsymbol{d})-\sigma_{t}^{2}|\boldsymbol{d} \boldsymbol{t}|^{2}, \\
& Q_{6}=-2 \psi(\boldsymbol{d} \cdot \boldsymbol{d})(\boldsymbol{d} \cdot \boldsymbol{s}), \\
& Q_{7}=2 \sigma_{t}^{2}|\boldsymbol{d} \boldsymbol{t}||\boldsymbol{d} \boldsymbol{s}|, \\
& Q_{8}=-\sigma_{t}^{2}|\boldsymbol{d} \boldsymbol{s}|^{2} .
\end{aligned}
$$

The maximum degree term (fourth-order term) is also the product of the square of each variable. The coefficients are determined by the input data as well. This result (elliptical form) agrees with the numerical simulations (Figure 3(c)).

\subsection{Relationship Between Algorithms-2 and -3}

The relationship between $\epsilon_{2}$ and $\epsilon_{3}$ can be derived using the one-to-one relationship between the algorithms represented by Equation (12). First, we solve Equation (12) for $\hat{\omega}_{2}$,

$$
\hat{\omega}_{2}=\frac{(1-\nu) \hat{\omega}_{3}}{1-\nu \hat{\omega}_{3}} .
$$

The relationship between the FVCs with propagated error can also be expressed by replacing $\hat{\omega}_{2}$ and $\hat{\omega}_{3}$ in Equation (48) with $\hat{\omega}_{2, \epsilon}$ and $\hat{\omega}_{3, \epsilon}$, respectively,

$$
\hat{\omega}_{2, \epsilon}=\frac{(1-\nu) \hat{\omega}_{3, \epsilon}}{1-\nu \hat{\omega}_{3, \epsilon}} .
$$

Using Equations (21) and (32), the above equation is rewritten as

$$
\hat{\omega}_{2}+\epsilon_{2}=\frac{(1-\nu)\left(\hat{\omega}_{3}+\epsilon_{3}\right)}{1-\nu\left(\hat{\omega}_{3}+\epsilon_{3}\right)} .
$$


Subtracting Equation (48) from Equation (50), we obtain the following relationship,

$$
\epsilon_{2}=\frac{(\nu-1) \epsilon_{3}}{\left(1-\nu \hat{\omega}_{3}\right)\left(\nu \hat{\omega}_{3}-1+\nu \epsilon_{3}\right)} .
$$

The relationship between $\epsilon_{2}$ and $\epsilon_{3}$ is one-to-one, as expected from Equation (12). Recall that the variable $\hat{\omega}_{3}$ is a function of the input data, and also that $\epsilon_{3}$ is a function of $\sigma_{t}$. Therefore, the variable $\epsilon_{2}$ is a function of both $\sigma_{t}$ and the input data. Note that this one-to-one relationship holds only when both algorithms use the same VI as a variable or a constraint. If the VIs used in the algorithms are different, the relationship is no longer one-to-one. The derivation of the relationship in such cases will be introduced later in this study.

The two propagated errors ( $\epsilon_{2}$ as $\left.\epsilon_{3}\right)$ should be positively correlated because the two FVC estimates $\left(\hat{\omega}_{2}\right.$ as $\left.\hat{\omega}_{3}\right)$ are positively correlated. To confirm this correspondence, we consider the partial derivative of $\epsilon_{2}$ with respect to $\epsilon_{3}$,

$$
\frac{\partial \epsilon_{2}}{\partial \epsilon_{3}}=\frac{1-\nu}{\left(\nu \hat{\omega}_{3}-1+\nu \epsilon_{3}\right)^{2}} .
$$

Since $\nu$ is lower than 1 [16], we have

$$
\frac{\partial \epsilon_{2}}{\partial \epsilon_{3}}>0
$$

confirming the positive correlation between $\epsilon_{2}$ and $\epsilon_{3}$. The derived result (one-to-one and positive correlation) agrees with the numerical example shown in Figure 3(d).

\section{Relationships Between Errors Propagated Within an Algorithm (Intra-Algorithm)}

Algorithms-2 (and -3) produce variable results, depending on the choice of two-band VI. For example, algorithm-2 in conjunction with NDVI as the endmember variable provides different values for FVC from that computed using the same algorithm but assuming SAVI as an endmember variable. Therefore, the propagated errors depend also on the choice of VI. This motivated us to derive the relationship between various errors propagated by algorithms- 2 or -3 (intra-algorithm relationships).

\subsection{Relationships Within Algorithm-2}

From Equation (22), the error propagated by algorithm-2 is written by

$$
\epsilon_{2}=\frac{\sigma_{t} \boldsymbol{a} \cdot \boldsymbol{e}}{\sigma_{t} \boldsymbol{b} \cdot \boldsymbol{e}+\phi}
$$

The error propagated by algorithm-2, assuming a different choice of VI $\left(\epsilon_{2}^{\prime}\right)$, is written as

$$
\epsilon_{2}^{\prime}=\frac{\sigma_{t} \boldsymbol{a}^{\prime} \cdot \boldsymbol{e}}{\sigma_{t} \boldsymbol{b}^{\prime} \cdot \boldsymbol{e}+\phi^{\prime}}
$$

where the two vectors $\boldsymbol{a}^{\prime}$ and $\boldsymbol{b}^{\prime}$ and the coefficient $\phi^{\prime}$ all depend on the VI choice, which is different from the result of Equation (54). The expressions of $\phi^{\prime}, \boldsymbol{a}^{\prime}$, and $\boldsymbol{b}^{\prime}$ can be defined using the VI values of the 
two endmember spectra, $v_{v}^{\prime}$ and $v_{s}^{\prime}$, and the coefficients of the VI for Equation (55), $\boldsymbol{c}_{1}^{\prime}, \boldsymbol{c}_{2}^{\prime}, r_{1}^{\prime}$, and $r_{2}^{\prime}$, as follows,

$$
\begin{aligned}
& \phi^{\prime}=\left(v_{v}^{\prime}-v_{s}^{\prime}\right)\left(\boldsymbol{c}_{2}^{\prime} \cdot \boldsymbol{\rho}_{t}+r_{2}^{\prime}\right)^{2} \\
& \boldsymbol{a}^{\prime}=\left(\boldsymbol{c}_{2}^{\prime} \cdot \boldsymbol{\rho}_{t}+r_{2}^{\prime}\right) \boldsymbol{c}_{1}^{\prime}-\left(\boldsymbol{c}_{1}^{\prime} \cdot \boldsymbol{\rho}_{t}+r_{1}^{\prime}\right) \boldsymbol{c}_{2}^{\prime} \\
& \boldsymbol{b}^{\prime}=\left(v_{v}^{\prime}-v_{s}^{\prime}\right)\left(\boldsymbol{c}_{2}^{\prime} \cdot \boldsymbol{\rho}_{t}+r_{2}^{\prime}\right) \boldsymbol{c}_{2}^{\prime}
\end{aligned}
$$

For the sake of brevity, we abbreviate the components of the four vectors used in the above equations as $\boldsymbol{a}=\left(a_{1}, a_{2}\right), \boldsymbol{b}=\left(b_{1}, b_{2}\right), \boldsymbol{a}^{\prime}=\left(a_{1}^{\prime}, a_{2}^{\prime}\right)$, and $\boldsymbol{b}^{\prime}=\left(b_{1}^{\prime}, b_{2}^{\prime}\right)$. A matrix representation of Equations (54) and (55) is given by

$$
\sigma_{t} X e=x
$$

where

$$
\begin{aligned}
& \boldsymbol{X}=\left[\begin{array}{ll}
b_{1} \epsilon_{2}-a_{1} & b_{2} \epsilon_{2}-a_{2} \\
b_{1}^{\prime} \epsilon_{2}^{\prime}-a_{1}^{\prime} & b_{2}^{\prime} \epsilon_{2}^{\prime}-a_{2}^{\prime}
\end{array}\right], \\
& \boldsymbol{x}=\left[\begin{array}{c}
-\phi \epsilon_{2} \\
-\phi^{\prime} \epsilon_{2}^{\prime}
\end{array}\right] .
\end{aligned}
$$

Equation (59) is solved for $\boldsymbol{e}$,

$$
\boldsymbol{e}=\frac{1}{\sigma_{t}} \boldsymbol{X}^{-1} \boldsymbol{x}
$$

where

$$
\begin{aligned}
& \boldsymbol{X}^{-1}=\frac{1}{|\boldsymbol{X}|}\left[\begin{array}{cc}
b_{2}^{\prime} \epsilon_{2}^{\prime}-a_{2}^{\prime} & -b_{2} \epsilon_{2}+a_{2} \\
-b_{1}^{\prime} \epsilon_{2}^{\prime}+a_{1}^{\prime} & b_{1} \epsilon_{2}-a_{1}
\end{array}\right],
\end{aligned}
$$

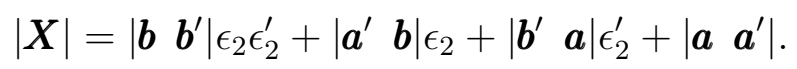

The actual form of $e$ becomes

$$
\boldsymbol{e}=\frac{1}{\sigma_{t}|\boldsymbol{X}|}\left[\begin{array}{c}
\left(\phi^{\prime} b_{2}-\phi b_{2}^{\prime}\right) \epsilon_{2} \epsilon_{2}^{\prime}+\phi a_{2}^{\prime} \epsilon_{2}-\phi^{\prime} a_{2} \epsilon_{2}^{\prime} \\
\left(\phi b_{1}^{\prime}-\phi^{\prime} b_{1}\right) \epsilon_{2} \epsilon_{2}^{\prime}-\phi a_{1}^{\prime} \epsilon_{2}+\phi^{\prime} a_{1} \epsilon_{2}^{\prime}
\end{array}\right]
$$

The relationships between $\epsilon_{2}$ and $\epsilon_{2}^{\prime}$ can be obtained by substituting Equation (65) into Equation (43). The final form becomes

$$
V_{1} \epsilon_{2}^{2}\left(\epsilon_{2}^{\prime}\right)^{2}+V_{2} \epsilon_{2}^{2} \epsilon_{2}^{\prime}+V_{3} \epsilon_{2}\left(\epsilon_{2}^{\prime}\right)^{2}+V_{4} \epsilon_{2}^{2}+V_{5}\left(\epsilon_{2}^{\prime}\right)^{2}+V_{6} \epsilon_{2} \epsilon_{2}^{\prime}+V_{7} \epsilon_{2}+V_{8} \epsilon_{2}^{\prime}+V_{9}=0
$$


where

$$
\begin{aligned}
& V_{1}=\left(\phi^{\prime} b_{2}-\phi b_{2}^{\prime}\right)^{2}+\left(\phi b_{1}^{\prime}-\phi^{\prime} b_{1}\right)^{2}-\sigma_{t}^{2}\left|\boldsymbol{b} \boldsymbol{b}^{\prime}\right|^{2}, \\
& V_{2}=2\left[\phi a_{2}^{\prime}\left(\phi^{\prime} b_{2}-\phi b_{2}^{\prime}\right)-\phi a_{1}^{\prime}\left(\phi b_{1}^{\prime}-\phi^{\prime} b_{1}\right)-\sigma_{t}^{2}\left|\boldsymbol{b} \boldsymbol{b}^{\prime} \| \boldsymbol{a}^{\prime} \quad \boldsymbol{b}\right|\right], \\
& V_{3}=2\left[\phi^{\prime} a_{1}\left(\phi b_{1}^{\prime}-\phi^{\prime} b_{1}\right)-\phi^{\prime} a_{2}\left(\phi^{\prime} b_{2}-\phi b_{2}^{\prime}\right)-\sigma_{t}^{2}\left|\boldsymbol{b} \boldsymbol{b}^{\prime} \| \boldsymbol{b}^{\prime} \boldsymbol{a}\right|\right] \text {, } \\
& V_{4}=\phi^{2}\left(a_{1}^{\prime}\right)^{2}+\phi^{2}\left(a_{2}^{\prime}\right)^{2}-\sigma_{t}^{2} \mid \boldsymbol{a}^{\prime} \quad \boldsymbol{b}^{2} \text {, } \\
& V_{5}=\left(\phi^{\prime}\right)^{2} a_{1}^{2}+\left(\phi^{\prime}\right)^{2} a_{2}^{2}-\sigma_{t}^{2}\left|\boldsymbol{b}^{\prime} \boldsymbol{a}\right|^{2},
\end{aligned}
$$

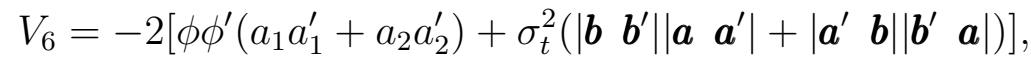

$$
\begin{aligned}
& V_{7}=-2 \sigma_{t}^{2}\left|\begin{array}{lll}
\boldsymbol{a}^{\prime} & \boldsymbol{b} \| \boldsymbol{a} & \boldsymbol{a}^{\prime}
\end{array}\right|
\end{aligned}
$$

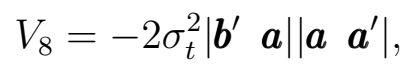

$$
\begin{aligned}
& V_{9}=-\sigma_{t}^{2}\left|\boldsymbol{a} \boldsymbol{a}^{\prime}\right|^{2} \text {. }
\end{aligned}
$$

This relationship becomes a fourth-degree polynomial, and the coefficients of the polynomial depend on the input data. However, the number of terms is different from the the number of terms in the relationships obtained in the previous section.

\subsection{Relationships Within Algorithm-3}

The intra-algorithm error relationships within algorithm-3 are almost the same as those described in the previous subsection. This is because the equation for $\epsilon_{3}$ can be obtained simply by substituting $\boldsymbol{a}, \boldsymbol{b}$, $\boldsymbol{a}^{\prime}, \boldsymbol{b}^{\prime}, \phi$, and $\phi^{\prime}$ with $\boldsymbol{s}, \boldsymbol{t}, \boldsymbol{s}^{\prime}, \boldsymbol{t}^{\prime}, \psi$, and $\psi^{\prime}$, respectively. The two vectors $\boldsymbol{s}^{\prime}$ and $\boldsymbol{t}^{\prime}$, and the coefficient $\psi^{\prime}$ are determined by the choice of VI, which is different from that for $\boldsymbol{s}, \boldsymbol{t}$, and $\psi$. The definitions of $\psi^{\prime}, \boldsymbol{s}^{\prime}$, and $\boldsymbol{t}^{\prime}$ are

$$
\begin{aligned}
\psi^{\prime} & =\left[\boldsymbol{c}_{1}^{\prime} \cdot \boldsymbol{\rho}_{t}+r_{1}^{\prime}+\eta^{\prime}\left(\boldsymbol{c}_{2}^{\prime} \cdot \boldsymbol{\rho}_{t}+r_{2}^{\prime}\right)\right]^{2}, \\
\boldsymbol{s}^{\prime} & =\zeta^{\prime}\left[\left(\boldsymbol{c}_{1}^{\prime} \cdot \boldsymbol{\rho}_{t}+r_{1}^{\prime}\right) \boldsymbol{c}_{2}^{\prime}-\left(\boldsymbol{c}_{2}^{\prime} \cdot \boldsymbol{\rho}_{t}+r_{2}^{\prime}\right) \boldsymbol{c}_{1}^{\prime}\right], \\
\boldsymbol{t}^{\prime} & =\left(\boldsymbol{c}_{1}^{\prime}+\eta^{\prime} \boldsymbol{c}_{2}^{\prime}\right)\left[\boldsymbol{c}_{1}^{\prime} \cdot \boldsymbol{\rho}_{t}+r_{1}^{\prime}+\eta^{\prime}\left(\boldsymbol{c}_{2}^{\prime} \cdot \boldsymbol{\rho}_{t}+r_{2}^{\prime}\right)\right],
\end{aligned}
$$

where

$$
\begin{aligned}
\eta^{\prime} & =-\frac{\boldsymbol{d} \cdot \boldsymbol{c}_{1}^{\prime}}{\boldsymbol{d} \cdot \boldsymbol{c}_{2}^{\prime}} \\
\zeta^{\prime} & =\frac{\left[\left(\boldsymbol{c}_{1}^{\prime} \cdot \boldsymbol{\rho}_{s}+r_{1}^{\prime}\right) \boldsymbol{c}_{2}^{\prime}-\left(\boldsymbol{c}_{2}^{\prime} \cdot \boldsymbol{\rho}_{s}+r_{2}^{\prime}\right) \boldsymbol{c}_{1}^{\prime}\right] \cdot \boldsymbol{\rho}_{v}+\left(r_{2}^{\prime} \boldsymbol{c}_{1}^{\prime}-r_{1}^{\prime} \boldsymbol{c}_{2}^{\prime}\right) \cdot \boldsymbol{\rho}_{s}}{\left(\boldsymbol{c}_{2}^{\prime} \cdot \boldsymbol{d}\right)^{2}} .
\end{aligned}
$$

The error propagated by algorithm-3 is given by

$$
\epsilon_{3}=\frac{\sigma_{t} s \cdot e}{\sigma_{t} t \cdot e+\psi}
$$

Similarly, the error propagated by the algorithm using the different VI is given by

$$
\epsilon_{3}^{\prime}=\frac{\sigma_{t} s^{\prime} \cdot \boldsymbol{e}}{\sigma_{t} \boldsymbol{t}^{\prime} \cdot \boldsymbol{e}+\psi^{\prime}}
$$


For brevity, the vectors in Equations (73) and (74) are abbreviated by $\boldsymbol{s}=\left(s_{1}, s_{2}\right), \boldsymbol{t}=\left(t_{1}, t_{2}\right), \boldsymbol{s}^{\prime}=$ $\left(s_{1}^{\prime}, s_{2}^{\prime}\right)$, and $\boldsymbol{t}^{\prime}=\left(t_{1}^{\prime}, t_{2}^{\prime}\right)$. Because all derivation steps are the same as those taken in the previous subsection, we simply summarize the final form.

$$
W_{1} \epsilon_{3}^{2}\left(\epsilon_{3}^{\prime}\right)^{2}+W_{2} \epsilon_{3}^{2} \epsilon_{3}^{\prime}+W_{3} \epsilon_{3}\left(\epsilon_{3}^{\prime}\right)^{2}+W_{4} \epsilon_{3}^{2}+W_{5}\left(\epsilon_{3}^{\prime}\right)^{2}+W_{6} \epsilon_{3} \epsilon_{3}^{\prime}+W_{7} \epsilon_{3}+W_{8} \epsilon_{3}^{\prime}+W_{9}=0
$$

where

$$
\begin{aligned}
& W_{1}=\left(\psi^{\prime} t_{2}-\psi t_{2}^{\prime}\right)^{2}+\left(\psi t_{1}^{\prime}-\psi^{\prime} t_{1}\right)^{2}-\sigma_{t}^{2}\left|\boldsymbol{t} \boldsymbol{t}^{\prime}\right|^{2}, \\
& W_{2}=2\left[\psi s_{2}^{\prime}\left(\psi^{\prime} t_{2}-\psi t_{2}^{\prime}\right)-\psi s_{1}^{\prime}\left(\psi t_{1}^{\prime}-\psi^{\prime} t_{1}\right)-\sigma_{t}^{2}\left|\boldsymbol{t} \boldsymbol{t}^{\prime}\right|\left|\boldsymbol{s}^{\prime} \boldsymbol{t}\right|\right] \text {, } \\
& W_{3}=2\left[\psi^{\prime} s_{1}\left(\psi t_{1}^{\prime}-\psi^{\prime} t_{1}\right)-\psi^{\prime} s_{2}\left(\psi^{\prime} t_{2}-\psi t_{2}^{\prime}\right)-\sigma_{t}^{2}\left|\boldsymbol{t} \boldsymbol{t}^{\prime}\right|\left|\boldsymbol{t}^{\prime} \boldsymbol{s}\right|\right] \text {, } \\
& W_{4}=\psi^{2}\left(s_{1}^{\prime}\right)^{2}+\psi^{2}\left(s_{2}^{\prime}\right)^{2}-\sigma_{t}^{2}\left|\boldsymbol{s}^{\prime} \boldsymbol{t}\right|^{2} \text {, } \\
& W_{5}=\left(\psi^{\prime}\right)^{2} s_{1}^{2}+\left(\psi^{\prime}\right)^{2} s_{2}^{2}-\sigma_{t}^{2}\left|\boldsymbol{t}^{\prime} \boldsymbol{s}\right|^{2} \text {, }
\end{aligned}
$$

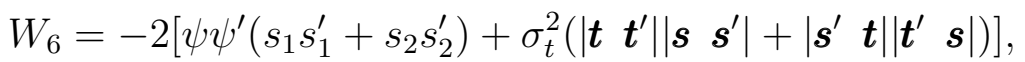

$$
\begin{aligned}
& W_{7}=-2 \sigma_{t}^{2}\left|\boldsymbol{s}^{\prime} \boldsymbol{t}\right|\left|\boldsymbol{s} \boldsymbol{s}^{\prime}\right| \text {, } \\
& W_{8}=-2 \sigma_{t}^{2}\left|\boldsymbol{t}^{\prime} \boldsymbol{s} \| \boldsymbol{s} \boldsymbol{s}^{\prime}\right| \text {, } \\
& W_{9}=-\sigma_{t}^{2}\left|s s^{\prime}\right|^{2} \text {. }
\end{aligned}
$$

This expression has the same characteristics as that of the intra-algorithm relationship for algorithm-2, with modifications to the coefficients.

\section{Inter-Algorithm Relationships With Different VI Assumptions}

The relationships between algorithms-2 and -3, derived in Section 5, are limited to the cases in which the same VI is chosen for both algorithms. In this section, we expand this limitation by deriving the relationship between two algorithms assuming different VIs. The derivation is basically the same as that described in Section 5, with different definitions of $\epsilon_{3}$. We replace $\epsilon_{3}$ with $\epsilon_{3}^{\prime}$ to indicate the different choice in VI for algorithm-3. The errors propagated by the algorithms are expressed by

$$
\begin{aligned}
\epsilon_{2} & =\frac{\sigma_{t} \boldsymbol{a} \cdot \boldsymbol{e}}{\sigma_{t} \boldsymbol{b} \cdot \boldsymbol{e}+\phi}, \\
\epsilon_{3}^{\prime} & =\frac{\sigma_{t} \boldsymbol{s}^{\prime} \cdot \boldsymbol{e}}{\sigma_{t} \boldsymbol{t}^{\prime} \cdot \boldsymbol{e}+\psi^{\prime}} .
\end{aligned}
$$

The final form of the relationship becomes

$$
Z_{1} \epsilon_{2}^{2}\left(\epsilon_{3}^{\prime}\right)^{2}+Z_{2} \epsilon_{2}^{2} \epsilon_{3}^{\prime}+Z_{3} \epsilon_{2}\left(\epsilon_{3}^{\prime}\right)^{2}+Z_{4} \epsilon_{2}^{2}+Z_{5}\left(\epsilon_{3}^{\prime}\right)^{2}+Z_{6} \epsilon_{2} \epsilon_{3}^{\prime}+Z_{7} \epsilon_{2}+Z_{8} \epsilon_{3}^{\prime}+Z_{9}=0
$$


where

$$
\begin{aligned}
& Z_{1}=\left(\psi^{\prime} b_{2}-\phi t_{2}^{\prime}\right)^{2}+\left(\phi t_{1}^{\prime}-\psi^{\prime} b_{1}\right)^{2}-\sigma_{t}^{2}\left|\boldsymbol{b} \boldsymbol{t}^{\prime}\right|^{2}, \\
& Z_{2}=2\left[\phi s_{1}^{\prime}\left(\psi^{\prime} b_{2}-\phi t_{2}^{\prime}\right)-\phi s_{1}^{\prime}\left(\phi t_{1}^{\prime}-\psi^{\prime} b_{1}\right)-\sigma_{t}^{2}\left|\boldsymbol{b} \boldsymbol{t}^{\prime} \| \boldsymbol{s}^{\prime} \boldsymbol{b}\right|\right], \\
& Z_{3}=2\left[\psi^{\prime} a_{1}\left(\phi t_{1}^{\prime}-\psi^{\prime} b_{1}\right)-\psi^{\prime} a_{2}\left(\psi^{\prime} b_{2}-\phi t_{2}^{\prime}\right)-\sigma_{t}^{2}\left|\boldsymbol{b} \boldsymbol{t}^{\prime}\right|\left|\boldsymbol{t}^{\prime} \quad \boldsymbol{a}\right|\right], \\
& Z_{4}=\phi^{2}\left(s_{1}^{\prime}\right)^{2}+\phi^{2}\left(s_{1}^{\prime}\right)^{2}-\sigma_{t}^{2}\left|\boldsymbol{s}^{\prime} \boldsymbol{b}\right|^{2}, \\
& Z_{5}=\left(\psi^{\prime}\right)^{2} a_{1}^{2}+\left(\psi^{\prime}\right)^{2} a_{2}^{2}-\sigma_{t}^{2}\left|\boldsymbol{t}^{\prime} \boldsymbol{a}\right|^{2},
\end{aligned}
$$

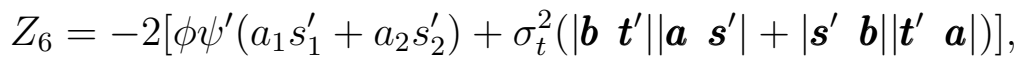

$$
\begin{aligned}
& Z_{7}=-2 \sigma_{t}^{2}\left|\boldsymbol{s}^{\prime} \quad \boldsymbol{b}\right|\left|\boldsymbol{a} \boldsymbol{s}^{\prime}\right| \\
& Z_{8}=-2 \sigma_{t}^{2}\left|\boldsymbol{t}^{\prime} \quad \boldsymbol{a}\right|\left|\boldsymbol{a} \boldsymbol{s}^{\prime}\right| \text {, } \\
& Z_{9}=-\sigma_{t}^{2}\left|\boldsymbol{a} \boldsymbol{s}^{\prime}\right|^{2} \text {. }
\end{aligned}
$$

Recall that the relationship between the error propagated by each algorithm is one-to-one if the same VI is assumed. However, the relationship is no longer one-to-one if different VIs are chosen for each algorithm, which is clearly indicated by Equation (79). (The equation represents an asymmetric ellipse.)

\section{Discussion and Conclusions}

This study presented derivations of the relationships among errors propagated in FVC using the three types of LMM-based algorithms. A band-correlated additive noise was assumed as the input error in the target reflectance spectrum. The additive noise depended on the directional parameter $(\theta)$ in the red-NIR reflectance space. The derivations proceeded by eliminating this parameter from the system of equations describe the relationships between the errors propagated in any combination of the two algorithms and their variations.

We found that the coefficients of the derived relationships depended on the input data for the algorithms, namely, the target spectrum, the endmember spectra, and the VI used in each algorithm, in addition to magnitude of the input error. We also found that the relationships could be represented by the same polynomial form, which corresponded to an asymmetric ellipse. One exception was found for the combination of algorithms-2 and -3. Specifically, when the two algorithms used an identical VI, the relationship between the two propagated errors became one-to-one. These results corresponded quite well with the numerically demonstrated relationships, which indicated the validity of our results.

From a practical point of view, algorithm selection is always a factor for successful analyses. A part of supportive information for the decision making is about the robustness against noises in a target spectrum. To provide such information a set of parameter studies would be required. In order to avoid such efforts the relationships between the propagated errors should be derived. Since this study provides a theoretical basis about the robustness of the three algorithms, it would eventually contribute to better algorithm selection. Further studies will be required to make the derived relationships be practical in the context of actual data processing and analysis.

The derived relationships provided information about the amplitude differences among the errors propagated in FVCs using the different algorithms. A comparison of the two algorithms showed that smaller amplitude propagated errors were indicative of a better algorithm. Therefore, our results can be 
used to select an optimal algorithm with respect to minimizing the propagated errors in FVCs. Moreover, because the coefficients of the derived relationships included only the magnitude of measurement errors as a model for input noise, the derived relationships could be used to avoid parameter studies of band-correlated information.

This study restricts the number of endmember spectra and the number of bands to two in order to facilitate the analytical discussions. We discussed only a limited number of algorithms under the framework of LMM. The limitations and restrictions should be expanded. The influence of the condition imposed in a form of VI is expected to be smaller as the number of endmember increases. In addition, analytical investigations of this kind would become extremely harder in such a case. However, the derived relationships clarify several important aspects of FVC retrieval using LMM-based algorithms.

\section{Acknowledgements}

This work was supported by JSPS KAKENHI 21510019 (HY).

\section{References}

1. Krinner, G.; Viovy, N.; de Noblet-Ducoudré, N.; Jérome, O.; Polcher, J.; Friedlingstein, P.; Ciais, P.; Sitch, S.; Prentice, I.C. A dynamic global vegetation model for studies of the coupled atmosphere-biosphere system. Global Biogeochem. Cycles 2005, 19, GB1015.

2. Jiang, Z.; Huete, A.R.; Chen, J.; Chen, Y.; Li, J.; Yan, G.; Zhang, X. Analysis of NDVI and scaled difference vegetation index retrievals of vegetation fraction. Remote Sens. Environ. 2006, $101,366-378$.

3. Jimenéz-Muñoz, J.C.; Sobrino, J.A.; Plaza, A.; Guanter, L.; Moreno, J.; Martinez, P. Comparison between fractional vegetation cover retrievals from vegetation indices and spectral mixture analysis: Case study of PROBA/CHRIS data over an agricultural area. Sensors 2009, 9, 768-793.

4. Barlage, M.; Zeng, X. The effects of observed fractional vegetation cover on the land surface climatology of the community land model. J. Hydrometeorol. 2004, 5, 823-830.

5. Zhang, J.; Walsh, J.E. Thermodynamic and hydrological impacts of increasing greenness in Northern High Latitudes. J. Hydrometeorol. 2006, 7, 1147-1163.

6. Wang, C.; Qi, J.; Cochrane, M. Assessment of tropical forest degradation with canopy fractional cover from Landsat ETM+ and IKONOS imagery. Earth Interact. 2005, 9, 1-18.

7. Xiao, J.; Moody, A. A comparison of methods for estimating fractional green vegetation cover within a desert-to-upland transition zone in central New Mexico, USA. Remote Sens. Environ. 2005, 98, 237-250.

8. Lobell, D.B.; Asner, G.P.; Law, B.E.; Treuhaft, R.N. Subpixel canopy cover estimation of coniferous forests in Oregon using SWIR imaging spectrometry. J. Geophys. Res. 2001, $106,5151-5160$.

9. Horwitz, H.M.; Nalepka, R.F.; Hyde, P.D.; Morgenstern, J.P. Estimating the Proportions of Objects within a Single Resolution Element of a Multispectral Scanner. In Proceedings of 7th International Symposium on Remote Sensing of Environment, Ann Arbor, MI, USA, May 17-21, 1971; pp. 1307-1320. 
10. Adams, J.B.; Smith, M.O.; Johnson, P.E. spectral mixture modeling: A new analysis of rock and soil types at the Viking Lander 1 Site. J. Geophys. Res. 1986, 91, 8098-8112.

11. Shimabukuro, Y.; Smith, J. The least-squares mixing models to generate fraction images derived from remote sensing multispectral data. IEEE Trans. Geosci. Remote Sens. 1991, 29, 16 -20.

12. Jasinski, M.F. Estimation of subpixel vegetation density of natural regions using satellite multispectral imagery. IEEE Trans. Geosci. Remote Sens. 1996, 34, 804-813.

13. Kallel, A.; Héarat-Mascle, S.L.; Ottlé, C.; Hubert-Moy, L. Determination of vegetation cover fraction by inversion of a four-parameter model based on isoline parametrization. Remote Sens. Environ. 2007, 111, 553 - 566.

14. Carpenter, G.A.; Gopal, S.; Macomber, S.; Martens, S.; Woodcock, C.E. A neural network method for mixture estimation for vegetation mapping. Remote Sens. Environ. 1999, 70, 138-152.

15. Guilfoyle, K.J.; Althouse, M.L.; Chang, C.-I. A quantitative and comparative analysis of linear and nonlinear spectral mixture models using radial basis function neural networks. IEEE Trans. Geosci. Remote Sens. 2001, 39, 2314-2318.

16. Obata, K.; Yoshioka, H. inter-algorithm relationships for the estimation of the fraction of vegetation cover based on a two endmember linear mixture model with the VI constraint. Remote Sens. 2010 , $2,1680-1701$.

17. Goward, S.N.; Markham, B.; Dye, D.G.; Dulaney, W.; Yang, J. Normalized difference vegetation index measurements from the advanced very high resolution radiometer. Remote Sens. Environ. 1991, 35, 257 - 277.

18. Miura, T.; Huete, A.; Yoshioka, H. Evaluation of sensor calibration uncertainties on vegetation indices for MODIS. IEEE Trans. Geosci. Remote Sens. 2000, 38, 1399 -1409.

19. Soffer, R.J.; Neville, R.A.; Staenz, K.; White, H.P. Sensitivity of Spectral Unmixing Analysis to a Spectrally Dependent Gain Error in Hyperspectral Data. In Proceedings of 2006 IEEE International Conference on Geoscience and Remote Sensing Symposium, Denver, CO, USA, July 31-August 4, 2006; pp. 1130-1133.

20. Gong, P.; Zhang, A. Noise effect on linear spectral unmixing. Ann. GIS 1999, 5, 52-57.

21. Barducci, A.; Mecocci, A. Theoretical and experimental assessment of noise effects on least-squares spectral unmixing of hyperspectral images. Opt. Eng. 2005, 44, 087008.

22. Wittich, K.P.; Hausing, O. Area-averaged vegetative cover fraction estimated from satellite data. Int. J. Biometeorol. 1995, 38, 209-215.

23. Carlson, T.N.; Ripley, D.A. On the relation between NDVI, fractional vegetation cover, and leaf area index. Remote Sens. Environ. 1997, 62, 241-252.

24. Gutman, G.; Ignatov, A. The derivation of the green vegetation fraction from NOAA/AVHRR data for use in numerical weather prediction models. Int. J. Remote Sens. 1998, 19, 1533-1543.

25. Carlson, T.N.; Arthur, S.T. The impact of land use-land cover changes due to urbanization on surface microclimate and hydrology: a satellite perspective. Global Planet Change 2000, $25,49-65$.

26. Lu, H.; Raupach, M.R.; McVicar, T.R. Decomposition of Vegetation Cover into Woody and Herbaceous Components Using AVHRR NDVI Time Series; Technical Report 35/01; CSIRO Land and Water: Canberra, ACT, Australia, 2001. 
27. Matsui, T.; Lakshmi, V.; Small, E.E. The effects of satellite-derived vegetation cover variability on simulated land-atmosphere interactions in the NAMS. J. Climate 2005, 18, 21-40.

28. Rouse, J.W., Jr.; Haas, R.H.; Schell, J.A.; Deering, D.W. Monitoring vegetation systems in the Great Plains with ERTS. In Third Earth Resources Technology Satellite-1 Symposium- Volume I: Technical Presentations; NASA SP-351; NASA: Washington, DC, USA, 1974; pp. 309-317.

29. Huete, A.R. A soil-adjusted vegetation index (SAVI). Remote Sens. Environ. 1988, 25, 295-309.

30. Jiang, Z.; Huete, A.R.; Didan, K.; Miura, T. Development of a two-band enhanced vegetation index without a blue band. Remote Sens. Environ. 2008, 112, 3833 - 3845.

31. Zeng, X.; Dickinson, R.E.; Walker, A.; Shaikh, M.; Defries, R.S.; Qi, J. Derivation and evaluation of global 1-km fractional vegetation cover data for land modeling. J. Appl. Meteorol. 2000, 39, 826-839.

32. Hirano, Y.; Yasuoka, Y.; Shibasaki, R. Pragmatic approach for estimation of vegetation cover ratio in urban area using NDVI. J. Jpn. Soc. Remote Sens. 2002, 22, 163-174, (in Japanese with English abstract).

33. Zhang, X.; Yan, G.; Li, Q.; Li, Z.L.; Wan, H.; Guo, Z. Evaluating the fraction of vegetation cover based on NDVI spatial scale correction model. Int. J. Remote Sens. 2006, 27, 5359-5372.

34. Okin, G.S.; Roberts, D.A. Linear Unmixing of Simulated, Noisy Spectra: Vegetation Detection Limits in Areas of Low Cover. In Proceedings of the 2000 AVIRIS Airborne Geoscience Workshop, Pasadena, CA, USA, February 23-25, 2000; p. 10.

(c) 2010 by the authors; licensee MDPI, Basel, Switzerland. This article is an open access article distributed under the terms and conditions of the Creative Commons Attribution license (http://creativecommons.org/licenses/by/3.0/). 